\title{
Involvement of cavernous sinus region by malignant neoplasms: Report of 5 cases
}

\author{
KHURSHID AHMAD, M.D., DLO \\ Detroit, Michigan \\ CHARLES YAMOKOSKI, D.O. \\ Largo, Florida \\ YOUNG H. KIM, M.D \\ Detroit, Michigan \\ M. JUDITH POST, M.D \\ Miami, Florida \\ JUAN V. FAYOS, M.D \\ Ann Arbor, Michigan
}

Case histories of 5 patients diagnosed as having involvement of the cavernous sinus by metastasis of a malignant tumor are described. In 2 cases, the tumors involved the cavernous sinus region either by direct extension or by invasion along the regional neurovascular structures from tumors of the head and neck area. In 3 cases, involvement was via hematogenous routes from distant sites. Each patient presented initially with signs of third, fourth, fifth, or sixth cranial nerve palsy. As demonstrated by computerized tomographic scanning, the cavernous sinus was the only intracranial site of involvement in all patients. A known history of malignancy and clinical signs suggestive of cavernous sinus involvement are the main diagnostic clinical criteria. Highresolution computed tomographic scanning with contrast enhancement is the radiographic procedure of choice. High doses of irradiation ( $>60 \mathrm{~Gy}$ ) appear to be most beneficial form of treatment for these patients. For patients who have clinical signs indicating a cavernous sinus mass too small to be detected by computed tomography, lower doses of radiation may be adequate.

The cavernous sinuses lie symmetrically alongside the body of the sphenoid bone in the middle cranial fossa. Their intricate relationships with several anatomic structures could produce well-defined syndromes when involved by a neoplastic process. The internal carotid artery and sixth cranial nerves enter the fibrous roof of the cavernous sinuses and run forward in its lateral wall. The fifth cranial nerve ganglia, with their ophthalmic and maxillary divisions, lie on the lateral wall of each sinus.

The cavernous sinus and adjacent structures could be involved by tumors of the head and neck region by direct extension of the neoplasm through the base of the skull., ${ }^{1,2}$ Ordinarily, this extension is from a nasopharyngeal carcinoma; it occurs later in the development of these tumors in almost onefourth of cases. ${ }^{3}$ Malignancy could also spread along the regional neurovascular structures. ${ }^{1-7}$ Alternatively, involvement could be through hematogenous routes ${ }^{1,2,8,9}$ from tumors located at a distant site. When there is involvement of the cavernous sinus by a tumor, the patient usually presents with palsy of the third, fourth, fifth, or sixth cranial nerves, because of the close anatomic relationship of these nerves to the cavernous sinus. Objective confirmation of a clinical diagnosis of cavernous sinus involvement has been difficult until recently, when computerized tomographic (CT) scanning of the brain with contrast enhancement has been found to be a major diagnostic tool. ${ }^{10}$

The basis of this paper is a review of a series of 5 cases with cavernous sinus involvement, which were evaluated at the Radiation Therapy Division of the University of Miami/Jackson Memorial Medical Center (UM/JMMC). The primary objectives were to determine the various routes of spread of tumor to the cavernous sinus and its relative frequency, the usefulness of CT scanning in diagnosing this condition, and whether early diagnosis and treatment with radiation therapy is beneficial.

\section{Report of cases}

During the period extending from October 1982 through February 1984, 2,170 new patients with diagnosis of ma- 
lignancy of various parts of the body were seen in the Radiation Therapy Division of UM/JMMC. Of these, 5 patients presented with symptoms and signs suggestive of cavernous sinus involvement. In all 5 cases, CT scans showed the cavernous sinus to be the only intracranial site of involvement.

\section{Case 1}

A 46-year-old black man was diagnosed as having T3 N2 M0 (stage IV) squamous cell carcinoma of the left tonsil. In April 1982, he underwent composite resection and left radical neck dissection. At the time of surgery, the left neck mass was found to involve the base of the skull and the internal carotid artery. Because of the extent of the tumor, it could not be removed completely. After adequate healing, the patient was started on a course of external irradiation with a $4 \mathrm{MV}$ linear accelerator. $\mathrm{He}$ received a total tumor dose of 66 Gy in 33 fractions over a period of 60 days. The cavernous sinus was out of the field of irradiation.

In October 1982, the patient complained of mild discomfort at the left supraorbital region and intermittent swelling on the left side of his face. Several weeks later, he was admitted to the hospital after suffering a seizure. Physical examination revealed left sixth cranial nerve palsy, periorbital edema of the left eye, and sensory deficit of the left face in the distribution of maxillary and mandibular divisions of the fifth cranial nerve. A CT scan of the brain demonstrated bony destruction at the base of the skull and an enhancing mass in the left cavernous sinus region (Fig. 1).

We consider this case to represent direct extension of neoplasm into the cavernous sinus. The patient was treated with chemotherapy only. We have been unable to assess the results of the therapy because the patient was lost to follow up.

\section{Case 2}

A 66-year-old white woman initially was diagnosed at another hospital in April 1981 as having basosquamous cell carcinoma of the left upper lip. At the time of surgery, the tumor was found to involve the left infraorbital nerve up to the infraorbital foramen. The mass was resected with narrow surgical margins. The patient received postoperative irradiation with $9 \mathrm{MeV}$ electron beam in another institution. The total dose delivered was $45 \mathrm{~Gy}$.

In July 1982, after several months of pain and swelling at the left cheek area, additional surgery was performed because of recurrence of the tumor. This time the mass was removed from infraorbital foramen back to foramen rotundum. On pathologic examination, the surgical margins of resection were free of tumor. The patient remained generally stable until January 1983, when CT scanning was performed to evaluate her complaint of persistent left frontal headache. It revealed a large, soft-tissue mass that extended from the level of infraorbital foramen into the cavernous sinus (Fig. 2). Radiation therapy was recommended to the patient, but she declined any further treatment.

We classify this case as a tumor extension along maxillary division of the fifth cranial nerve to the cavernous sinus region.

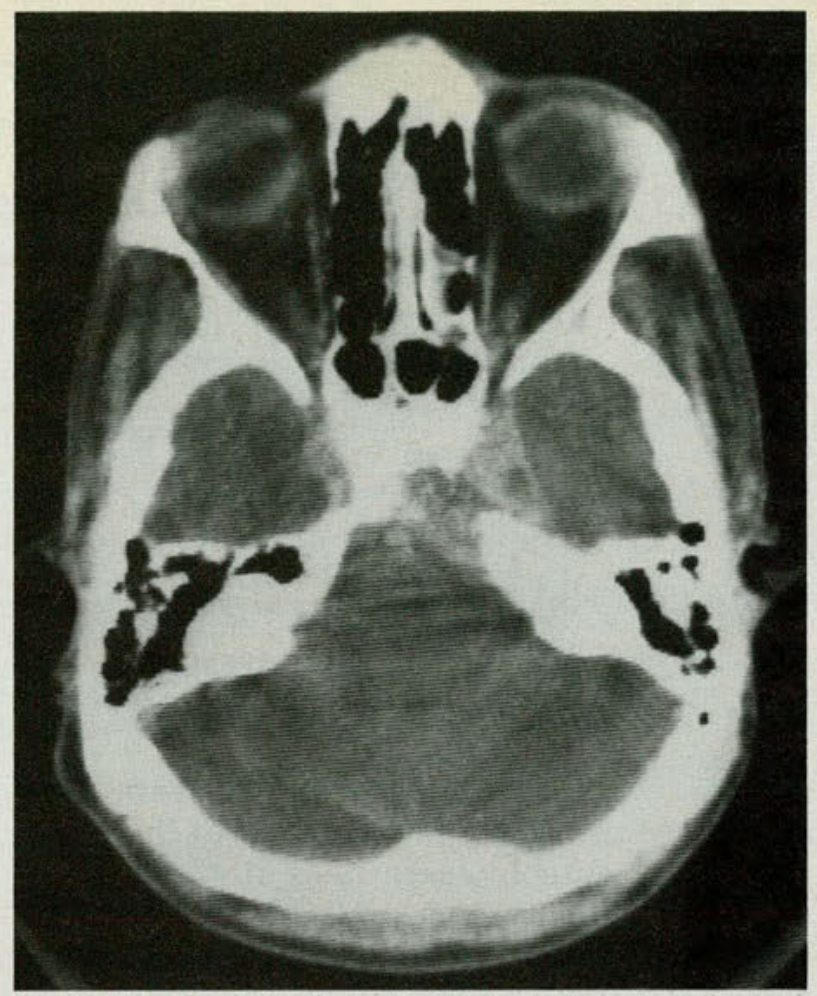

Fig. 1. CT scan of patient 1 demonstrates bone destruction of the base of the skull in the left cavernous sinus region.

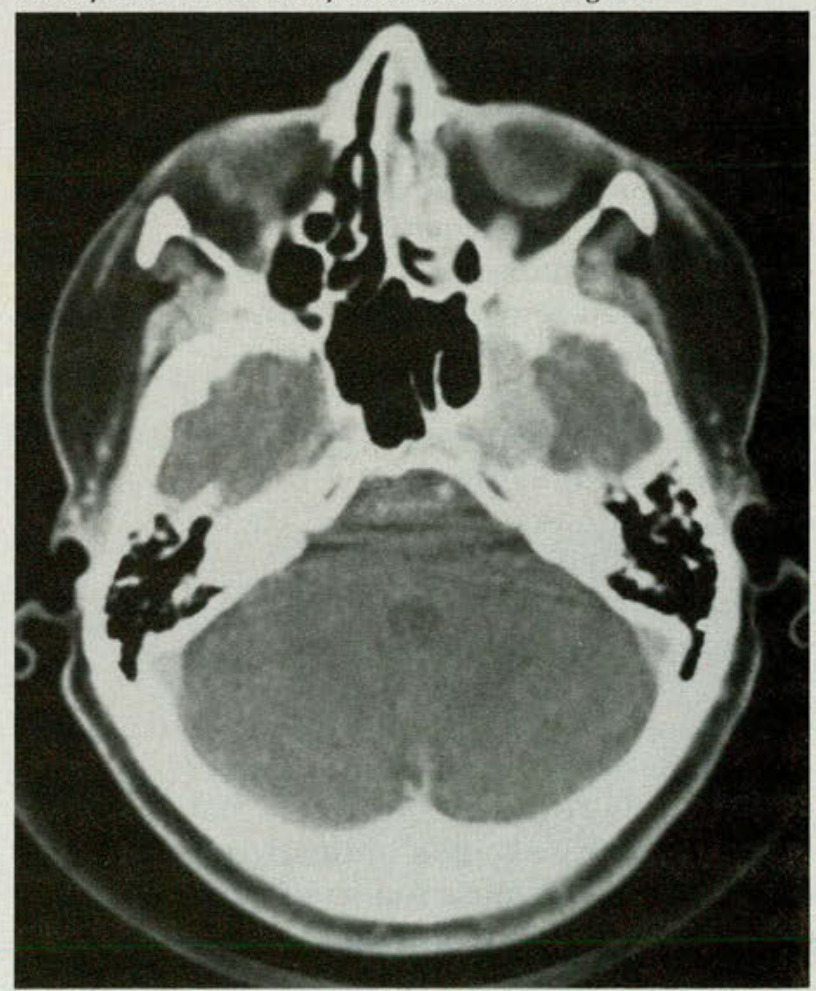

Fig. 2. Case 2 CT scan shows a large, enhancing mass at the left infraorbital foramen that involves the left cavernous sinus.

\section{Case 3}

A 42-year-old white woman underwent left modified radical mastectomy and left axillary node dissection in April 1982. Her disease was reported pathologically as infil- 
trating ductal carcinoma of the breast with metastasis to multiple axillary lymph nodes. After surgery, she was maintained on a chemotherapy regimen consisting of cyclophosphamide, methotrexate, 5-fluorouracil, vincristine, and prednisone. She was progressing well until June 1983, when tumor recurred on the left anterior chest wall. Bone scanning demonstrated increased uptake in both the skull and the right pelvis, which indicated metastatic disease. The chest wall was treated with irradiation, which controlled the tumor locally.

During October 1983, the patient suddenly developed diplopia. On physical examination, she was found to have palsy of the left sixth cranial nerve. A CT brain scan was generally inconclusive of any gross pathologic findings. There was, however, a subtle increased density of the left cavernous sinus region (Fig. 3 ). On the basis of progressive neurologic symptoms, the patient was offered radiation therapy to the cavernous sinus area, but she refused. With respect to the left sixth cranial nerve symptoms, her progress was monitored by an ophthalmologist, who prescribed a course of oral steroids.

The patient developed complete ptosis of the left upper eyelid, blurred vision, and complete paralysis of the left sixth cranial nerve in January 1984 . Repeat CT scanning demonstrated an enhancing lesion in the left cavernous sinus region (Fig. 4). The patient was now agreeable to proceed with localized irradiation of the involved site, which was started on a $4 \mathrm{MV}$ linear accelerator. Through opposing lateral ports, she received $51 \mathrm{~Gy}$ in 17 fractions over a duration of 23 days. At follow-up assessment, she had complete resolution of the left sixth cranial nerve palsy, and there was only minimal residual ptosis of the upper eyelid. We consider this case to represent metastasis to the cavernous sinus from a remote site.

\section{Case 4}

A 70-year-old white man was diagnosed in June 1982 as having poorly differentiated squamous cell carcinoma of the right supraglottic region. It was classified as T3 N3 M0 (stage IV) tumor. The patient had bilateral neck node metastasis, with the largest node measuring $9.8 \mathrm{~cm}$. on the right side and a mass at the left jugulodigastric region measuring $3 \times 3 \mathrm{~cm}$. During June and July 1982, he was treated with a combination of external radiation and biweekly local hyperthermia with a $915 \mathrm{MHz}$ microwave unit. He received 70 Gy from a 4 MV linear accelerator through opposing lateral ports in 35 fractions over a period of 50 days. The right neck mass received an additional boost of $4 \mathrm{~Gy}$. Toward the end of irradiation, there was only minimal residual tumor at the primary site. There was complete regression of the left neck mass, while the right neck mass regressed to about 60 percent of its original size. In October 1982, the patient presented with sudden blindness of the left eye and paresthesia over the left cheek. Examination revealed complete ophthalmoplegia of the left eye and ptosis of the left upper eyelid. A CT scan revealed an enhancing mass in the left cavernous sinus and orbital apex, without any demonstrable tumor at the base of the skull. The right cavernous sinus was unremarkable. The metastatic work up also confirmed lung and liver spread.

The patient was started on systemic chemotherapy and

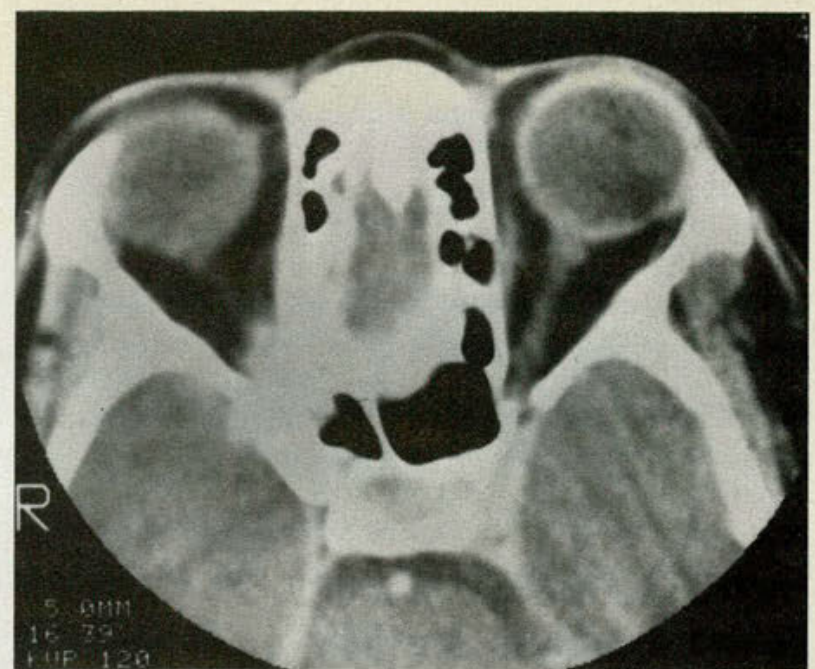

Fig. 3. Questionable increased density of the left cavernous sinus is seen in this $C T$ scan from patient 3 .

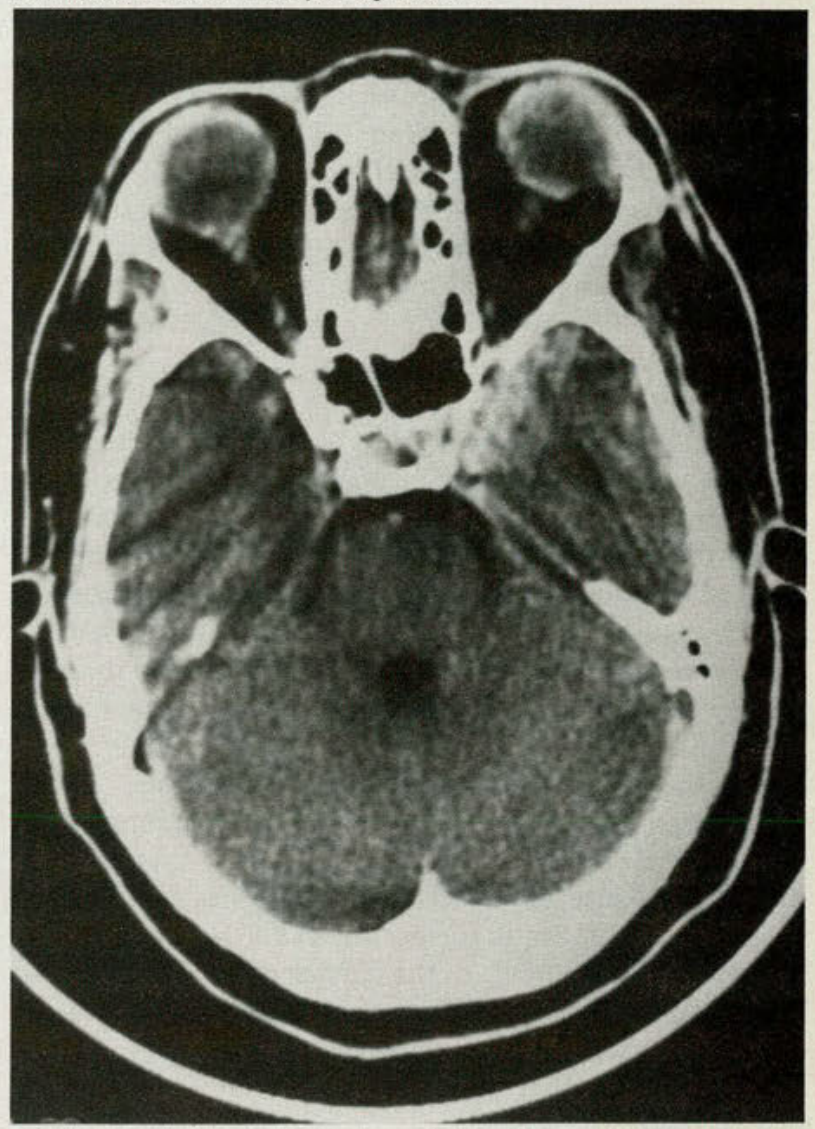

Fig. 4. In case 3, repeat CT scan obtained 3 months after Figure 3 demonstrates an enhancing mass in the left cavernous sinus.

local irradiation to the cavernous sinus region. Two days into therapy, he developed ptosis of the right upper eyelid. Repeat CT scanning did not demonstrate any gross involvement of the right cavernous sinus region. The patient left the country after receiving $22 \mathrm{~Gy}$ in 11 fractions. At that point, there was marked improvement of the ptosis on the right side and slight improvement on the left side. This case represents hematogenous spread of a supraglottic carcinoma to the cavernous sinus. 


\section{Case 5}

A 64-year-old black woman was diagnosed as having an infiltrating ductal carcinoma of the right breast in October 1981 . She left the country after the diagnosis without having received any treatment. In February 1983, she returned to the hospital complaining of intense pain in the right arm, hoarseness, numbness of the right forehead of 2 months' duration, and decreased right visual acuity of 3 weeks' duration. Physical examination revealed paralysis of the left vocal cord, decreased tactile sensation over the right forehead, ptosis of the right upper eyelid, and paralysis of the third, fourth, and sixth cranial nerves on the right. She had a mass in the upper outer quadrant of the right breast and multiple enlarged right axillary and supraclavicular lymph nodes.

A CT scan of the brain revealed a soft-tissue mass in the region of the right cavernous sinus. The patient was treated with chemotherapy (cyclophosphamide, Adriamycin, and 5-fluorouracil) and irradiation to the cavernous sinus region. She received $42 \mathrm{~Gy}$ in 14 fractions over a period of 19 days. At the completion of irradiation, there was no clinical improvement in the cranial nerve involvement. The patient continued to demonstrate progression of systemic disease despite the chemotherapy. This case represents involvement of cavernous sinus by hematogenous metastatic disease from a distant primary site.

\section{Analysis of cases}

The incidence of cavernous sinus as the only intercranial site of involvement is low. Only 5 such patients were seen in our department during a 17 -month period. All patients had a diagnosis of carcinoma originating from various parts of the body, and all of them presented with clinical features suggestive of tumor spread to the cavernous sinus region. In cases 1 and 2 , the tumor spread directly to the cavernous sinus, through the bones of the base of the skull in the first case and along the maxillary division of the fifth cranial nerve in the second case. In the other 3 patients, the involvement was via hematogenous routes. In all patients, the cavernous sinus was the only intracranial site of involvement that was documented radiographically by high-resolution CT scanning using contrast enhancement. In the earlier stages of disease in case 3 and on the right side in case 4 , CT scanning failed to demonstrate any tumor mass in the cavernous sinus area despite clinical signs suggestive of cavernous sinus involvement.

Three patients (cases 3,4 , and 5) received irradiation of the cavernous sinus region. There was marked improvement of the neurologic deficit in case 3 . This patient had received 51 Gy in 17 fractions over a period of 23 days. Patient 4, who had clinical suspicion of right cavernous sinus involvement without any radiographic evidence of mass in the region, also showed marked improvement of ptosis of the right eye, despite somewhat lower doses of irradiation ( 22 Gy in 11 fractions). Because of a large tumor mass on the left, this patient showed no improvement on that side with the same dose. There was also no improvement of clinical signs in patient 5 , who received $42 \mathrm{~Gy}$ in 14 fractions.

\section{Discussion}

Primary malignant tumors of the cavernous sinus are rare. Tumors in this region occur because of direct extension or invasion from a carcinoma in the head and neck region or from a remote site through a hematogenous route. ${ }^{1-9}$ Access to the cavernous sinus for tumors of the head and neck region is thought to be via extension along the neurovascular structures or by direct tumor extension that has destroyed the adjoining anatomic areas. ${ }^{1-3}$ Cases 1 and 2 are examples of local spread of tumor through the base of the skull bones and along the neurovascular structures, respectively, while the spread was via hematogenous routes in the rest of the patients. The involvement of cavernous sinus from nasopharyngeal carcinoma, which used to be a common occurence, ${ }^{3}$ is now an uncommon event in our experience, presumably because of our routine inclusion of cavernous sinuses in the irradiation ports.

Physical examination as well as high-resolution CT scanning, as recommended by Kline and associates, ${ }^{10}$ played an important role in diagnosis. The CT scans, however, failed to diagnose tumor in the early stages of disease despite the clinical features suggestive of cavernous sinus involvement in case 3 and on the right side in case 4.

A review of the literature reveals limited information with respect to definitive irradiation of tumors in this region; the majority of articles are in reference to benign diseases. ${ }^{11-13}$ One article ${ }^{14}$ presented prior to the era of supervoltage radiation recommended doses in the range of 40-100 Gy for cavernous sinus involvement. Only 3 of our patients received cavernous sinus radiation therapy after establishment of clinical and radiographic diagnosis. One patient (case 3 ), who had radiographic evidence of a mass in the cavernous sinus, showed marked improvement in the neurologic deficit after a dose of 51 Gy in 17 fractions (daily dose of $3 \mathrm{~Gy}$ ). Using conventional fractionation, the biologic effect of this dose is equivalent to $64 \mathrm{~Gy}$ in 32 fractions (daily dose of $2 \mathrm{~Gy}$ ). No neurologic improvement was noticed in patient 5 and on the left side in patient 4 , both of whom received doses less than 50 Gy. Patient 4, who developed clinical signs of right cavernous sinus involvement (unconfirmed by CT scan), showed marked improvement in ptosis of the right eye despite a low dose of $22 \mathrm{~Gy}$.

These findings suggest that small doses of irradiation to the cavernous sinus region are beneficial in cases in which metastasis is possible, although still radiographically undetectable. It is, therefore, important to diagnose the condition early and to treat the cavernous sinus with radiation to achieve maximum regression in certain patients: those who 
have both clinical and radiographic evidence of cavernous sinus involvement by a tumor; and those in whom the disease is microscopic and cannot be detected by radiographic means but who meet the criteria of having a known diagnosis of malignancy, and signs suggestive of cavernous sinus involvement, and in whom no other etiologic factors can be found. Once there is radiographic evidence of a mass, higher doses of radiation in excess of $60 \mathrm{~Gy}$ with a conventional fractionation regimen, as received by patient 3 , are necessary to achieve benefit.

\section{Summary}

Case histories of 5 patients are presented and the various routes of cavernous sinus involvement by tumors originating from different parts of the body are discussed. Physical examination and high-resolution CT scanning played an important role in these patients' diagnosis. It appears that early diagnosis, early initiation of treatment, and high doses of radiation ( $>60 \mathrm{~Gy}$ ), particularly when the mass is large, are necessary to control the tumor in this region. Moderate doses can be beneficial in cases when clinical symptoms are present but the tumor is so small that it cannot be detected by present CT scanning techniques.

\footnotetext{
1. Rucker, C.W.: The causes of paralysis of the third, fourth and sixth cranial nerves. Am J Opthalmol 61:1293-8, May 66

2. Jefferson, G.: The Bowman Lecture. Concerning injuries, aneurysms and tumors involving the cavernous sinus. Trans Ophthalmol 73:117-52, 1953

3. del Regato, J.A., and Spjut, H.J.: Cancer of the respiratory system and upper digestive tract. In Ackerman and del Regato's Cancer: Diagnosis,
}

treatment and prognosis. C.V. Mosby Co., St. Louis, 1977, pp. 301-18 4. Moore, C.E., Hoyt, W.F., and North, J.B.: Painful ophthalmoplegia following treated squamous cell carcinoma of the forehead. Orbital apex involvement from centripetal spread via the supraorbital nerve. Med $\mathrm{J}$ Aust 1:657-9, 1 May 76

5. Dodd, G.D., et al.: The dissemination of tumors of the head and neck via the cranial nerves. Radiol Clin N Am 8:445-61, Dec 70

6. Ballantyne, A.J., et al.: Extension of cancer of the head and neck through peripheral nerves. Am J Surg 106:651-67, Oct 63

7. Unsold, R., et al.: Metastatic infiltration of nerves in the cavernous sinus. Arch Neurol 37:59-61, Jan 80

8. Mills, R.P., et al.: Bilateral cavernous sinus metastasis and opthalmoplegia. J Neurosurg 55:463-6, Sep 77

9. Thomas, J.E., and Yoss, R.E.: The parasellar syndrome. Problems in determining etiology. Mayo Clin Proc 45:617-23, Sep 70

10. Kline, L.B., et al.: The cavernous sinus. A computed tomographic study. AJNR 2:299-305, Jul 81

11. Walker, J.R., and Hall, C.D.: Pituitary adenoma presenting as recurring painful ophthalmoplegia. Surg Neurol 8:319-22, Nov 77

12. Mackay, A., and Hosobuchi, Y.: Treatment of intracavernous extensions of pituitary adenoma. Surg Neurol 10:377-83, Dec 78

13. Trobe, J.D., Glaser, J.S., and Post, J.D.: Meningiomas and aneurysms of the cavernous sinus. Arch Opthalmol 96:457-67, Mar 78

14. Godtfredsen, E.: Ophthalmo-neurological symptoms in connection with malignant nasopharyngeal tumors. Br J Ophthalmol 31:78-100, Feb 47

Accepted for publication in September 1986. Updating, as necessary, has been done by the authors.

Dr. Ahmad is an associate professor of radiology, Wayne State University School of Medicine, Detroit, Michigan. Dr. Yamokoski is a staff radiologist, Sun Coast Hospital, Largo, Florida. Dr. Kim is an associate professor of radiology at WSUSM. Dr. Post is professor of radiology, at the University of Miami School of Medicine, Miami, Florida. Dr. Fayos is a staff radiation oncologist, St. Joseph's Hospital, Ann Arbor, Michigan.

Dr. Ahmad, Radiation Oncology Research and Development Center, Wayne State University School of Medicine, $4201 \mathrm{St}$. Antoine, Detroit, Michigan 48201. 


\section{American Osteopathic Association \\ Continuing Medical Education}

\section{CERTIFICATION OF HOME STUDY}

This is to certify that I,

activity for AOA CME credits.

Please print

completed the following

Type of activity (such as reading or listening)

Name of journal(s) or audio-tape and date(s) of issue(s):

(One-half CREDIT may be granted for each issue or tape)

MAIL TO: AOA Division of CME, 212 East Ohio Street, Chicago, Illinois 60611

KEEP A DUPLICATE FOR YOUR RECORDS!

The Home Study form is intended to document individual reading of recognized scientific journals, listening to approved audio-tapes, and other approved home study courses and programs under the criteria described for Category 2-B.

Only one type of home study, such as reading, should be indicated on a single form, though multiple issues of scientific journals may be listed.

This form should not be used, however, when CME quiz cards for the AOA Journal are submitted separately.

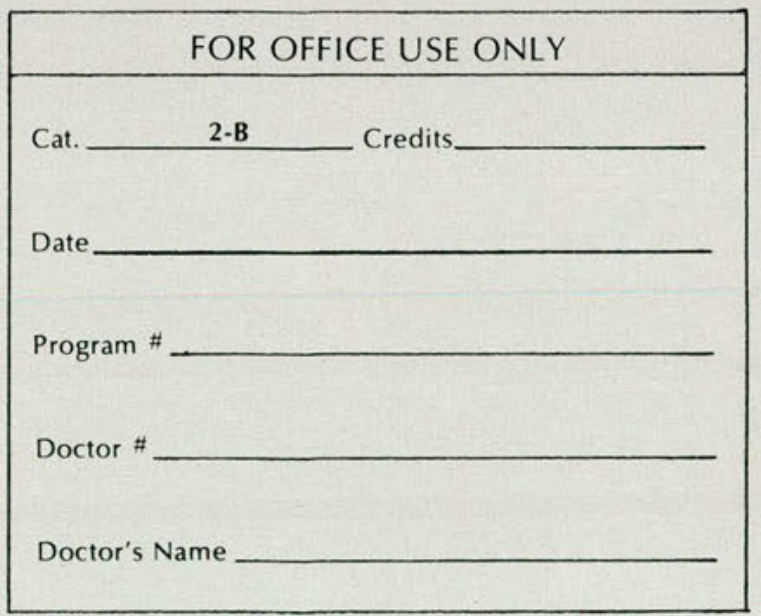

Please refer to the revised CME GUIDE for additional information. 